\title{
ESTABLISHING A GLOBAL NETWORK OF ORCHID SEED BANKS
}

\author{
PhiLIP T. SEATON \\ Jodrell Laboratory, Royal Botanic Gardens, Kew, Richmond, Surrey, TW9 3DS, U.K. \\ philipseaton@googlemail.com
}

KeY WoRDS: orchid, seed, storage, seed banks

\section{Introduction}

Although it would appear that very few orchid species have, as yet, become extinct in the wild, such are the threats to many orchid populations, this situation appears unlikely to continue (Koopowitz, 2001). Just as significant as the loss of individual species is the erosion of genetic diversity within those species. As numbers decline the size of the gene pool reduces, and the rarer alleles begin to disappear. Thus, not only is it important to preserve individual representative specimens, but also the greater genetic variation contained within populations. Seed banks can provide a means of preserving this genetic diversity in a minimum space and at a minimum cost (Linington \& Pritchard, 2001). They also have the added benefit of encouraging the raising of orchids from seed, thereby reducing the pressure on wild populations from unscrupulous collectors.

The idea of setting up an orchid seed banking network is not new. It was proposed in 1984 at the World Orchid Conference in Miami (Greatwood, 1984). Despite Lewis Knudson's findings in the 1950 's that dry seeds of at least some orchid species could be stored for at least 20 years at refrigerator temperatures (Knudson, 1954), the view nevertheless persisted in some circles at that time that orchid seeds were short-lived. Detailed research over the past twenty years has, however, demonstrated that orchid seeds are no more short-lived than seeds of some other plant families (Pritchard et al. 1999). As far as we can tell, the vast majority should remain viable for decades (Seaton \& Pritchard, 2003) at the very least when stored dry and at low temperatures. They can therefore be descibed as being, broadly speaking, orthodox. Orthodox seed is seed that can be dried to low moisture contents, and therefore maintained in storage at below-freezing temperatures. There are a few minor differences in the behaviour of orchids to typical orthodox seeds at some sub-zero temperatures (around -30 to -50 C) where, contrary to expectations, longevity is reduced (Pritchard et al. 1999). These temperatures are, however, easily avoided, and dry orchid seed can be stored successfully at either $5 \mathrm{C}$, the temperature of a domestic refrigerator, or $-20 \mathrm{C}$, the temperature of a domestic deep freeze (Seaton \& Pritchard, 2003).

\section{Seed Collection and Seed Qualit}

Orchid seeds are covered both by the environmental laws of the host countries, as well as by international regulations. Before any seed is collected, permission must be obtained from the relevant authorities. Likewise, seed from cultivated plants must be derived from legally collected plants.

Accurate identification of the parent plant is essential. If the seed bank is attached to a botanical garden a flower can be preserved in spirit, and deposited in the garden's herbarium. An alternative solution would be to submit a good quality photograph of a flower, and perhaps one of the whole plant in flower too. Happily digital photography and scanners make it relatively easy to include such information in a database.

Once permission has been obtained, the next step is the collection of a representative sample of good quality seed. Put simply, you could not reasonably expect poor quality seed to maintain high levels of viability for as long as good quality seed. Quality depends upon a number of variables. These include the age of the flower (and therefore, most likely, of the pollen) at the time of pollination, timing of harvest, parentage, and environmental conditions during maturation of the seed capsule.

In the case of greenhouse-grown plants, the tempta- 
tion is to enjoy the flowers for as long as possible before pollinating them. However, the older the flower the older the pollen, and old pollen is itself likely to be less viable. To produce seed of optimum quality, the best advice is probably to pollinate the flowers as soon as they are fully open. Large, strong, well-grown plants, tend to produce more viable seeds than their less robust partners.

Although sowing of immature embryos (so-called 'green pod' techniques) has advantages in terms of reducing the time a capsule is carried by the parent plant, avoiding problems of surface-sterilization of seeds during the sowing procedure and, in the case of some 'hardy' species, circumventing dormancy mechanisms, the likelihood is that such seed cannot be stored as successfully as mature seed. As seeds mature within the capsule they gradually acquire the ability to survive drying, particularly towards the end of the maturation process. Drying is a key component of successful orchid seed storage. Seed is therefore best harvested at, or just prior to, splitting of the seed capsule. This implies a prior knowledge of the phenology of the species in question, and frequent observation as the capsule matures. In order to find the plant once more when out of flower it may be necessary to used coloured tags, or a metal detector if metal tags are employed. Seed should be collected in paper envelopes.

If outcrossing typically leads to more vigorous offspring, the converse is also generally true - selfing, or repeated inbreeding leads to less vigorous offspring. Thus there is strong evidence of inbreeding depression in Restrepia, both in terms of a reduction in the percentage of viable seeds produced and the a reduction in vigour of the seedlings (Millner et al. 2007 in progress).

Collection of seed samples in dry environments is likely to be a much easier task than that in the humid tropics, where contamination by fungi and bacteria may be a serious problem. Indeed, a more practical option may be to raise plants specifically for seed collection in living collections with their advantages of enabling controlled pollinations and the ability to monitor seed capsule development.

Once harvested, seeds should be examined to check their viability. Ideally a sample can be examined under low magnification using a microscope, but a x10 hand lens will often be sufficient for at least a cursory examination. Potentially viable seed can be recognised by the presence of a plump embryo (Seaton \& Ramsay, 2005). Although this is, in itself, no guarantee that the seed will germinate; it may require a special medium or a compatible symbiotic fungus or, in some instances, a dormancy-breaking procedure; the presence of such an embryo in freshly harvested seeds generally suggests the seed is viable. In addition to the obviously full embryos, some seed coats will house somewhat reduced embryos. Others will contain no embryos at all.

\section{Drying Seed to a Suitable Moisture Content}

The benefits of reducing seed moisture content are, if anything, greater than those of reducing seed storage temperature. At one extreme, moist seed will support the growth of fungal and bacterial spores, which will multiply rapidly and kill the embryo; infected seed soon become almost impossible to sterilize and to sow in a sterile flask without contamination. At the other end of the spectrum, life processes depend on the presence of moisture. Thus, reducing seed moisture contents to the extreme can dramatically shorten life-spans.

The aim is to obtain a seed moisture content as close to the optimum as is practical (neither too high, nor not too low). If placed in a humid atmosphere, seed will gradually absorb moisture. Likewise, in a dry atmosphere seed will lose water to the air. In either case, over a period of time, the seed and the atmosphere will reach an equilibrium; at which point moisture is neither lost nor gained. The seed will have achieved its equilibrium moisture content.

Where basic laboratory facilities are available a saturated solution of calcium chloride is recommended (lithium chloride might be even better, but there may be problems with availability). A saturated solution of calcium chloride gives a relative humidity around 30 $\%$ at $20 \mathrm{C}$, and seed moisture contents of 4 to $6 \%$. The saturated solution should occupy at least one quarter of the volume of the desiccator (Seaton \& Pritchard, 2003). As long as some undissolved salt remains in the solution, the relative humidity will remain constant whilst the container remains at that temperature. 


\section{An alternative desiccant}

For the amateur or hobbyist, who may have difficulty accessing calcium chloride, dried rice is a suitable alternative. Toasted rice has been used as a desiccant for a wide range of both temperate and tropical seeds (Sadik \& White, 1982; Akromah \& BennettLartey, 1996). Any supermarket brand will do. Simply spread the rice as a thin layer (no more than one or two grains thick) in the bottom of a baking tray, and dry in the oven at around $100 \mathrm{C}$ or slightly higher overnight.

It is important to remember that the rice will require regular regeneration as, with repeated use, it will itself gradually become increasingly moist. The drying capacities (i.e. how much moisture it is capable of absorbing) of rice is also generally unknown, so you should use plenty of it, filling the desiccator at least three quarters full of dried rice (Seaton \& Ramsay, 2005). Dried rice may also be considered the ideal desiccant for use in the field. Where there is a long time interval between harvest and reaching the seed storage facilities, the freshly-harvested seed can be placed in a suitable container in a small desiccator containing dried rice, and then dried to a more optimal seed moisture content on returning to the laboratory.

\section{A word about Silica Gel}

As with dried rice, unless it is regenerated each time it is used, silica gel slowly absorbs moisture from the atmosphere, its water absorbing capacity gradually declines, and it produces a different, higher, relative humidity.

A second, and potentially serious, problem with using dry silica gel as a desiccant is that it can produce very low moisture contents indeed: so low that they are potentially damaging to the embryo and actually reduce seed longevity (Seaton \& Pritchard, 2003). The use of silica gel as a desiccant, although its use for short-term storage may be acceptable, is not recommended for long term storage.

\section{Storage Temperature}

Good quality (high initial germination) dry orchid seed stored in air-tight vessels at a suitable seed moisture content, will maintain its viability at a tempera- ture of $5 \mathrm{C}$ in a domestic refrigerator for many years. Further reducing storage temperature from refrigerator temperature to the temperature of a domestic freezer (around -18 to $-20 \mathrm{C}$ ) leads to additional increases in seed longevity.

\section{Storage containers}

Many people store seeds in paper envelopes. Waxed paper will not take up moisture, and the seed does not stick to it. For long periods of storage (a number of years), however, hermetically sealed tubes are preferable. Glass tubes are preferable to plastic (where seeds tend to adhere to the sides of the tubes). Seeds should be stored in tubes with a volume that provides a minimum of head space above the seeds. Keeping the volume of air in the head space to a minimum when compared to the volume occupied by the seeds means that the seeds will completely dominate the system. This avoids the potential problem of seeds equilibrating to a new and different moisture content within the storage tube during transfer of that tube to the storage room or facility. If the volume of air is kept to a minimum then the moisture within the seed will overwhelm any effect of the moisture content of the surrounding atmosphere.

A potential problem arises with the long-term integrity of any seal. Storage jars, with their combination of a natural rubber seal and a clamp have been demonstrated to be the best available option (Manger et al. 2003). At the Millennium Seed Bank Project (MSBP) at Kew the additional precaution is taken of renewing the seals at ten year intervals.

Tubes can be stored within storage jars. After equilibration to a suitable moisture content, seeds are placed within hermetically-sealed tubes. Sachets of blue silica gel can be included to act as an indicator (not as a desiccant) of the performance of seals if any air leaks occur. The aim is to enable us to judge if moist air leaking past the seal into the jar. If it is, the seed should be re-dried and the seal should be replaced.

Every time a tube of seeds is opened the seed will begin to re-equilibrate with the moisture in the atmosphere. There is a choice to be made between re-equilibrating the remainder of the seed lot over an appropriate constant humidity solution before returning to 
storage, or storing seed lots in a number of individual tubes which will be opened once only. If the intention is to remove seed samples for sowing at regular intervals, the latter option may be the most appropriate as long as the seed lot is thoroughly mixed at the start, ensuring that as far as possible, each tube is representative of the whole seed lot.

\section{Maintaining a collection}

There is more to establishing a seed bank than simply placing the seed in storage and forgetting about it in the safe and secure knowledge that it will still be there for future generations to draw on if and when necessary. Upon arrival at the seed storage facility, the first step is that of cleaning the seed, separating it from the dry tissue of the seed capsule, usually by gentle sieving. Seed germination should then be tested immediately prior to storage, and then at regular (say 10 year) intervals to monitor seed viability. This does two things, it flags up the necessity for regeneration, and contributes to the pool of knowledge.

It is often the simple things that cause the problems. Good record keeping is vital. It is important to label everything at the outset to avoid any possibility of mix-ups. Tubes, packets and jars should all be labelled and dated, and details kept in a note-book and/or on a computer spreadsheet. All the above information needs to be recorded on a suitable database. Species, photograph, provenance, name of donor, date of harvest, date of receipt, percentage germination upon receipt, medium used to germinate the seed - all need to be recorded for future access. None of this can happen without secure, long-term funding for seed storage facilities.

Two types of collection are generally recognised. The first, an active collection, where accessions are withdrawn at regular intervals. A number of shortterm seed banks already exist in the world of the hobbyist grower and, because the seed is stored for a few years at the most, these are active seed banks. Such seed banks are suitable for supplying seed for botanical gardens, researchers, hobbyist and commercial growers

In a long-term account, seed is maintained untouched in storage for many years as a reserve, with material only removed if and when there is a need to regenerate plant material for the seed bank. A seed collection should be viewed as a living entity. However long-lived, plants in living collections do have finite life-spans and need to be replaced at regular intervals.

Seed banks can be viewed in the same way as conventional money banks - there will be withdrawals as well as deposits. When an accession is withdrawn we ought to be able to say two things about the seed lot. First, we should be able to say that the seed is viable. There is no point in sending out dead seed, and indeed there may be little point if the viability is very low. Second, we should say what medium the seed can be germinated on. After all, different media will give different percentage germinations. All media are not equally suitable for all species.

\section{What should we store?}

Much as we would like to store everything, it would seem wise to establish a list of priorities. Red Lists would appear to be the ideal tool for assessing the conservation status of species, unfortunately there are currently very few orchid species on the 2004 Global Red List. The Conference of the Parties to the CBD adopted the the Global Strategy for Plant Conservation (GSPC) at its meeting in The Hague in 2002. The ultimate and long-term objective of the GSPC is to halt the current and continuing loss of plant diversity through 16 outcome-orientated global targets for 2010. Target 8 is of particular relevance to ex situ plant conservation: 60 per cent of threatened plant species in accessible ex situ collections, preferably in the country of origin, and 10 per cent of them included in recovery and restoration programmes. If we are to have any chance of meeting the targets we need to begin storing orchid seed immediately. We simply cannot afford to wait until the desired Red Lists are completed.

\section{Conclusion}

We are engaged in a race against time. If we don't act quickly, much of the world's diversity, including orchids, will not be available for future generations to enjoy. The setting up of a global network of orchid seed banks is a cost-effective way of meeting this challenge. 


\section{LiterATURE Cited}

Akromah, R. \& S.O. Bennett-Lartey. 1996. Seed drying over toasted rice. Tropic. Sc. 36:32-33.

Greatwoood, J. 1984. Extract from the proceedings of the International Orchid Commission, March, 1984. Amer. Orch. Soc. Bull. 53: 737.

Knudson, L. 1954. Storage and viability of orchid seed. Amer. Orch. Soc. Bull. 22: 260-260.

Koopowitz, H. 2001. Orchid and their Conservation. B.T. Batsford Ltd., London.

Linington, S.H. \& H.W. Pritchard. 2001. Gene Banks. Pp.165-181 in: S.A. Levin (editor-in-chief). Encyclopedia of Biodiversity. Vol. 3. Academic Press, New York.

Manger, K.R., J. Adams \& R.J. Probert. 2003. Selecting Seed Containers for the Millennium Seed Bank Project: a technical review and survey. Pp. 637-652 in: R.D.

Millner, H.J., T.C. Baldwin \& P.T. Seaton. 2007. A botanical review of the genus Restrepia (Orchidaceae) and its conservation.
Pritchard, H.W., A.L.C. Poynter \& P.T. Seaton. 1999. Interspecific variation in orchid seed longevity in relation to ultra-dry storage and cryopreservation. Lindleyana. 14: 92-101.

Sadik, S. \& J.W. White. 1982. True potato seed drying over rice. Potato Res. 25: 269-272.

Seaton, P.T. \& H.W. Pritchard. 2002. Recent developments in orchid seed banking. Pages 390-396 in J. Clark, W.M. Elliot, G. Tingley \& J. Biro, editors. Proceedings of the $16^{\text {th }}$ World Orchid Conference April 1999, Vancouver. The Vancouver Orchid Society. Richmond BC. Canada.

Seaton, P.T. \& H.W. Pritchard. 2003. Orchid germplasm collection, storage and exchange. Pp, 227-258 in: K.W. Dixon, P. Cribb, S. Kell \& R. Barrett (eds.). Orchid Conservation. Natural History Publications (Borneo), Kota Kinabalu, Sabah.

Seaton, P.T. \& M.M. Ramsay. 2005. Growing Orchids from Seed. Royal Botanic Gardens, Kew, UK.

Previously a biology lecturer, Philip Seaton now devotes himself full-time to orchid conservation. He is Secretary of Orchid Conservation International and the Orchid Specialist group, and runs a micropropagation laboratory at a local school. A past editor of The Orchid Review, he has written around one hundred popular and scientific articles on a wide range of orchid topics. He has illustrated and co-authored Growing Orchids from Seed. He received the degree of Master of Philosophy for his research into orchid seed storage, and is currently working to promote the establishment of a global network of orchid seed banks. 